\title{
ANALYTIC FUNCTIONS WITH UNIVALENT DERIVATIVES AND ENTIRE FUNCTIONS OF EXPONENTIAL TYPE
}

\author{
BY S. M. SHAH
}

ABstract. Functions $f$, analytic and univalent in the unit disc, and such that all successive derivatives $f^{(k)}$ are univalent in this disc, are necessarily transcendental entire functions of exponential type. These functions, and functions $f$ having an infinite number of derivatives $f^{\left(n_{k}\right)}$ univalent in the unit disc, are discussed. Entire functions of bounded index are of exponential type and their properties are also discussed.

1. Introduction. Let $f(z)$ be analytic in the unit disc $D:|z|<1$. We say that $f$ is univalent in $D$ if for each pair of distinct points $z_{1}, z_{2}$ in $D$, $f\left(z_{1}\right) \neq f\left(z_{2}\right)$. In $\$ \$ 1-4$ we give a brief survey of functions analytic and ${ }^{1}$ univalent in $D$. Functions $f$ such that $f(z)$ and each successive derivative $f^{(k)}(z)$ are univalent in $D$ are considered next in $\$ 5$. Such functions $f$ must be transcendental entire functions of exponential type. Related problems of functions $f$ such that $f(z)$ and a sequence of derivatives $f^{\left(n_{k}\right)}(z)$ are univalent or of functions $f$ such that $f(z)$ is entire and $f^{(k)}(z)$ is univalent in $|z|<\rho_{k}\left(\rho_{k}>0\right)$ are considered in $\$ \$ 6-10$. This is followed by a section (\$11) on multivalent functions and three sections (\$\$12-14) on functions of bounded index. An entire function $f(z)$ is said to be of bounded index if there exists an integer $N$, independent of $z$, such that

$$
\max _{0 \leqq s \leqq N}\left\{\frac{\left|f^{(s)}(z)\right|}{s !}\right\} \geqq \frac{\left|f^{(j)}(z)\right|}{j !},
$$

for $j=1,2, \ldots$ and for all $z$. The smallest such integer $N$ is called the index of $f$. An entire function $f$ of bounded index $N$ is of exponential type not exceeding $(N+1)$. Finally we mention some unsolved problems.

2. Conditions for the univalence of $f$. Let

$$
f(z)=\sum_{0}^{\infty} a_{n} z^{n}, \quad|z|<1 .
$$

\footnotetext{
An expanded version of an invited address delivered to the 669th meeting of the American Mathematical Society at the University of Louisiana, Baton Rouge on November 22, 1969 ; received by the editors September 15, 1971. Research supported by NSF Grant GP-19533.

AMS 1970 subject classifications. Primary 30A32, 30A34, 30A64, 30A66; Secondary 30A04, 40C05, 40C15.

Key words and phrases. Entire functions, exponential type, univalent, transcendental entire, bounded index, Schwarzian derivative, Koebe function, starlike, close-to-convex, convex, order and lower order, slowly oscillating, natural boundary, central index, Whittaker constant, mean $p$-valent, areally mean $p$-valent, complete metric space, first category, summability methods, matrix transformation.
}

${ }^{1}$ In this article we shall not consider meromorphic univalent functions. 
If $a_{1} \neq 0$ and

$$
\sum_{2}^{\infty} n\left|a_{n}\right| \leqq\left|a_{1}\right|
$$

then $f$ is analytic and univalent in $D$ and continuous on the closure of $D$. To prove this, let $z_{1}, z_{2} \in D, \max _{i=1,2}\left|z_{i}\right|=r<1, z_{1} \neq z_{2}$. Then

$$
\begin{aligned}
\left|\frac{f\left(z_{2}\right)-f\left(z_{1}\right)}{z_{2}-z_{1}}\right| & =\left|a_{1}+\sum_{2}^{\infty} a_{n}\left(z_{2}^{n-1}+z_{2}^{n-1} z_{1}+\cdots+z_{1}^{n-1}\right)\right| \\
& \geqq\left|a_{1}\right|-\sum_{2}^{\infty} n\left|a_{n}\right| r^{n-1}>0 .
\end{aligned}
$$

This implies that $f$ is univalent in $D$. Further, for every $N \geqq 1$,

$$
\sum_{0}^{N}\left|a_{n}\right| \leqq\left|a_{0}\right|+\left|a_{1}\right|+\sum_{2}^{\infty} \frac{n\left|a_{n}\right|}{n} \leqq\left|a_{0}\right|+\frac{3\left|a_{1}\right|}{2}
$$

and continuity of $f$ follows.

If the radius of convergence of the series in (2.1) defining $f$ is $R$, then $f$ is univalent in $|z|<\rho \leqq R$, if $a_{1} \neq 0$ and

$$
\sum_{n=2}^{\infty} n\left|a_{n}\right| \rho^{n-1} \leqq\left|a_{1}\right|
$$

Let $f$ be analytic in $D$. If $f$ is univalent in $D$ then $f^{\prime}(z) \neq 0$ in $D[32$, p. 23]. If

$$
\operatorname{Re}\left(a f^{\prime}(z)\right)>0, \quad z \in D,
$$

for some complex number $a,|a|=1$, then $f$ is univalent in $D$. This follows immediately from the following integral expression

$$
\operatorname{Re}\left\{\frac{a\left(f\left(z_{2}\right)-f\left(z_{1}\right)\right)}{z_{2}-z_{1}}\right\}=\int_{0}^{1} \operatorname{Re}\left\{a f^{\prime}\left((1-w) z_{1}+w z_{2}\right)\right\} d w .
$$

Another criterion for univalence of $f([62]$; see also [29]) is as follows. Let

$$
\{w, z\}=\left(\frac{f^{\prime \prime}}{f^{\prime}}\right)^{\prime}-\frac{1}{2}\left(\frac{f^{\prime \prime}}{f^{\prime}}\right)^{2}
$$

be the Schwarzian derivative of $w=f(z)$ with respect to $z$. In order that $w=f(z)$ be univalent in $D$ it is necessary that

$$
|(w, z)| \leqq 6 /\left(1-|z|^{2}\right)^{2}
$$

and sufficient that

$$
|(w, z)| \leqq 2 /\left(1-|z|^{2}\right)^{2} .
$$


Becker [2] has recently proved that $f$ is univalent in $D$ if

$$
\left|\frac{f^{\prime \prime}(z)}{f^{\prime}(z)}\right| \leqq \frac{1}{\left(1-|z|^{2}\right)} .
$$

3. Class $S$. Let $S$ denote the collection of functions $f$ analytic and univalent in $D$ and normalized by the conditions $f(0)=0, f^{\prime}(0)=1$. Thus $f \in S$ can be written as

$$
f(z)=z+\sum_{2}^{\infty} a_{n} z^{n}, \quad|z|<1 .
$$

Bieberbach [6] proved in 1916 that, for $f \in S$,

$$
\left|a_{2}\right| \leqq 2
$$

with equality if and only if

$$
f(z)=K_{\alpha}(z) \equiv z /\left(1-z e^{i \alpha}\right)^{2} \quad(\alpha \text { real }) .
$$

This function $K_{\alpha}$ (Koebe function) maps $D$ on the whole plane slit radially from $w=-\frac{1}{4} e^{-i \alpha}$ to infinity. It is extremal not only for $a_{2}$ but also for a number of other problems. Since $\left|a_{n}\right|=n, n=2,3, \ldots$ for this function $K_{\alpha}$, it was conjectured that, for $f \in S$,

$$
\left|a_{n}\right| \leqq n, \quad n=2,3, \ldots,
$$

with equality only for the Koebe function. This conjecture, called the Bieberbach conjecture, was proved for $n=3$ by Loewner [58] in 1923, for $n=4$ by Charzynski and Schiffer ([18]; see also [30]) in 1960, and for $n=6$ by Pederson [66] in 1968 and Ozawa [64] in 1969 independently of each other. Garabedian and Schiffer [31] proved that (3.4) holds for a function $f \in S$ which is "close enough" to the Koebe function and Aharonov has shown (3.4) to hold if $\left|a_{2}\right|<0.867$ ([1]; see also [9]).

For each fixed $f \in S$, Hayman (see [38, pp. 112-113]) has shown that $\left|a_{n}\right| \leqq n\left(n>n_{0}(f)\right)$. For all $n \geqq 2$, Littlewood proved in 1925 (see [38, p. 10]) that $\left|a_{n}\right|<e n$. This estimate has recently been improved to $\left|a_{n}\right|<1.081 n(n \geqq 2)$ by Carl H. Fitzgerald (see also [32, p. 612]).

4. Subclasses of $S$. A function $f \in S$ is said to be starlike univalent in $D$, or briefly starlike in $D$ if $f(D)$ is starlike with respect to the origin $w=0$. A necessary and sufficient condition for $f \in S$ to be starlike in $D$ is that [63, pp. 220-222], [38, pp. 14-16],

$$
\operatorname{Re}\left(z f^{\prime}(z) / f(z)\right)>0, \quad|z|<1 .
$$

We shall denote this subclass of functions by $S^{*}$. From (4.1) it is easy to obtain, for $f \in S^{*}$, the following integral representation formula 


$$
z \frac{f^{\prime}(z)}{f(z)}=\frac{1}{2 \pi} \int_{0}^{2 \pi} \frac{1+z e^{-i t}}{1-z e^{-i t}} d V(t)
$$

where $V(t)$ is an increasing function of $t, V(t)-t$ has period $2 \pi$ and $(1 / 2 \pi) \int_{0}^{2 \pi} d V(t)=1$. A second subclass of $S$ is the class of convex univalent functions. We say that $f \in S$ is convex univalent in $D$ if $f(D)$ is a convex set. We denote this subclass of $S$ by $K$. A necessary and sufficient condition for $f \in S$ to be in $K$ is that [38, pp. 140-141], [32, p. 166],

$$
\operatorname{Re}\left(1+z f^{\prime \prime}(z) / f^{\prime}(z)\right)>0, \quad|z|<1 .
$$

If $f \in K$ then $\left|a_{n}\right| \leqq 1$. If $f \in S^{*}$ then $\left|a_{n}\right| \leqq n$.

A third subclass of functions is the class of close-to-convex functions introduced by Kaplan [46]. A function $f \in S$ is close-to-convex if and only if

$$
\operatorname{Re}\left(f^{\prime}(z) / \phi^{\prime}(z)\right)>0, \quad|z|<1,
$$

where $\phi(z) / \phi^{\prime}(0) \in K$. (If $f$ is analytic in $D$ and satisfies the close-to-convex condition (4.4) then it is univalent.) For this class (3.4) also holds. If $f$ is defined by (2.1) and satisfies (2.2) and if $f(0)=0, f^{\prime}(0) \neq 0$, then $f$ is starlike in $D$ [33]. From this we can conclude that if [33]

$$
\sum_{k=2}^{\infty} k^{2}\left|a_{k}\right| \leqq\left|a_{1}\right|
$$

then $f$ is convex in $D$.

For more information on various problems of univalent function theory we refer the reader to five excellent survey articles by Bernardi [3], Hayman [39], Goluzin [32, pp. 577-628], Goodman [35] and Robertson [73]. We list some recent papers in the bibliography at the end and refer to an exhaustive bibliography by Bernardi [4], for books and periodical literature up to 1965 .

5. Functions with univalent derivatives. Let $f \in S$ and let $E$ denote the subclass

$$
E=\left\{f \mid f \in S, f^{(k)} \text { is univalent in } D \text { for } k=1,2, \ldots\right\} .
$$

If $f \in E$ then $f$ must be a transcendental entire function of exponential type, that is,

$$
\limsup _{r \rightarrow \infty} \frac{\log M(r, f)}{r} \equiv T^{*}<\infty
$$

where as usual $M(r, f)=\max _{|z|=r}|f(z)|$. (Note that functions, for which $0 \leqq T^{*}<\infty$, and in particular functions of order less than one, are all 
functions of exponential type.) More precisely we have [84]

$$
|f(z)| \leqq \frac{\exp (2 \alpha|z|)-1}{2 \alpha},
$$

where $\alpha=\sup \left\{\left|a_{2}\right|: f \in E\right\}$ and

$$
\pi / 2 \leqq \alpha<1.7208 \text {. }
$$

To prove this we note that if $f \in E$ then $a_{n+1} \neq 0$. Define $F_{n}$ in $D$ by

$$
F_{n}(z)=\frac{f^{(n)}(z)-n ! a_{n}}{(n+1) ! a_{n+1}} .
$$

Then $F_{n} \in E$ and we have

$$
\left|a_{n+2}\right| \leqq \frac{2 \alpha\left|a_{n+1}\right|}{n+2} .
$$

An inductive argument gives $\left|a_{n}\right| \leqq(2 \alpha)^{n-1} / n !(n \geqq 2)$. This implies that $f$ is entire and satisfies (5.3). Since $\left|a_{2}^{2}-a_{3}\right| \leqq 1-\{M(1)\}^{-2}$ ([44], [88]), we have

$$
\alpha^{2} \leqq 3\left(1-4 \alpha^{2} /\left(e^{2 \alpha}-1\right)^{2}\right) .
$$

This implies the right-hand inequality in (5.4). To complete the proof of (5.4) we observe that $\phi(z)=(\exp (\pi z)-1) / \pi \in E$ and $a_{2}$ for this function is $\pi / 2$.

We note here that the property of univalence is only one of the properties which forces $f$ to be entire. Consider a property (A) which a function analytic in $D$ is able to possess. We say that $(\mathrm{A})$ is an admissible property provided the following hold: (i) if $f$ has (A) then $f^{\prime}(0) \neq 0$. (ii) If $f$ has (A) and if $b$ and $c$ are complex numbers with $b \neq 0$, then the function $F(z)=b f(z)+c$ also has (A). Let $T$ be the family of functions $f$, analytic in $D$, of the form (3.1). Let $T(A)$ be the subclass of $T$ such that if $f \in T(A)$ then $f^{(n)}$ has property (A) for $n=0,1,2, \cdots$. Suppose that $T(A)$ is not empty and let $\alpha_{A}=\sup \left\{\left|a_{2}\right|: f \in T(A)\right\}$. If $\alpha_{A}<\infty$ and $f \in T(A)$ then $f$ is a transcendental entire function of exponential type not greater than $2 \alpha_{A}$ [86]. For instance one can take property (A) to be property (K). We say that $f$ has $(\mathrm{K})$ if $f$ is convex univalent in $D$. Then $(\mathrm{K})$ is an admissible property. Further $\alpha_{K}=\sup \left\{\left|a_{2}\right|: f \in T(K)\right\}$ lies between $\frac{1}{2}$ and 0.6838 [86].

6. Not all derivatives univalent. Let $f$ be defined in $D$ by (2.1) and let $\left\{n_{k}\right\}_{1}^{\infty}$ be a sequence of strictly increasing positive integers. Suppose that each $f^{\left(n_{k}\right)}$ is univalent in $D$. Let $R$ be the radius of convergence of the series in (2.1). If the sequence $\left\{n_{k}\right\}$ does not increase very rapidly, we may have $R>1$. Thus, for instance [86], 


$$
\liminf _{k \rightarrow \infty}\left(n_{1} \cdots n_{k}\right)^{1 / n_{k}} \leqq R \limsup _{k \rightarrow \infty} 4^{k / n_{k}} \leqq 4 R .
$$

From (6.1) it is easy to show that if $n_{k+1}-n_{k}=o(\log k)$ then $R=\infty$ and $f$ is entire. If $n_{k+1}-n_{k}=O(1)$ then $f$ is of exponential type.

A more general result of this type is as follows. Let $\phi(x)$ and $\theta(x)$ be two slowly oscillating functions (see [86] and the references given there) and let $1 \leqq \phi(k) \leqq n_{k}-n_{k-1} \leqq \theta(k)$ for $k=2,3, \cdots$. If each $f^{\left(n_{k}\right)}$ is univalent in $D$ and

$$
\limsup _{k \rightarrow \infty} \frac{\theta(k) \log \theta(k)}{\phi(k) \log k}=\alpha<1,
$$

then $f$ is an entire function of order not greater than $1 /(1-\alpha)$.

If however the sequence $\left\{n_{k}\right\}$ increases very rapidly, say

$$
n_{k+1} \geqq n_{k} \log n_{k} \log \log n_{k},
$$

then $R$ may not exceed unity. In fact there exists [86] a function $f$, analytic in $D$ and an increasing sequence of positive integers $\left\{n_{k}\right\}_{k=1}^{\infty}$ such that $f$ and each $f^{\left(n_{k}\right)}$ map $D$ univalently onto convex domains and yet the unit circle is the natural boundary of $f$.

7. Derivatives with varying radii of univalence. Let $\rho(f)$ be the largest number with the property that $f$ is analytic and univalent in an open disc about the origin of radius $\rho$. We shall write $\rho\left(f^{(n)}\right)=\rho_{n}$. Suppose now that $f$ is defined by $f(z)=\sum_{0}^{\infty} a_{n} z^{n}$. Let $R$ denote the radius of convergence of this series. Then we have [85]

$$
\liminf _{n \rightarrow \infty} n \rho_{n} \leqq 4 R
$$

and

$$
R \log 2 \leqq \limsup _{n \rightarrow \infty} n \rho_{n} .
$$

If $\left|a_{n-1} / a_{n}\right|$ is ultimately a nondecreasing sequence, then

$$
R \log 2 \leqq \liminf _{n \rightarrow \infty} n \rho_{n} \leqq \limsup _{n \rightarrow \infty} n \rho_{n} \leqq 4 R .
$$

Thus (a) if $f$ is a transcendental entire function then $\lim \sup _{n \rightarrow \infty} n \rho_{n}=\infty$, and (b) if $\lim _{n \rightarrow \infty} n \rho_{n}=\infty$, then $f$ is a transcendental entire function. (See also [85, Theorem 3].) The converse of (a) is false. There exists a function $f$ analytic in the unit disc and in no larger disc $|z|<R$, where $R>1$, such that $\lim \sup n \rho_{n}=\infty$. The converse of (b) is also false [85].

8. Radii of univalence and entire functions. Let $f$ be a transcendental entire function of order $\Lambda$ and lower order $\lambda$ (see $[8$, p. 8]). When $0<\Lambda$ $<\infty$, let $T=\lim \sup _{r \rightarrow \infty} \log M(r) / r^{\Lambda}$ denote the type and $t=$ 
$\lim \inf _{r \rightarrow \infty} \log M(r) / r^{\Lambda}$ denote the lower type. The following theorems are due to Boas, Pólya and Takenaka respectively.

THEOREM A [7]. If $f(z)$ is a transcendental entire function and if

$$
T^{*}=\limsup _{r \rightarrow \infty} \frac{\log M(r)}{r}<\log 2,
$$

then there is a sequence $\left\{n_{p}\right\}_{p=1}^{\infty}$ such that $\rho_{n_{p}}=\rho\left(n_{p}\right) \geqq 1$ for all $p$.

Levinson [56] supplied a second proof of this. Boas also pointed out that, if $T^{*}=0$, then

$$
\limsup _{n \rightarrow \infty} \rho_{n}=\infty .
$$

THEOREM B [67]. If $f(z)$ is a transcendental entire function of order $\Lambda$, then

$$
\liminf _{n \rightarrow \infty} \frac{\log \rho_{n}}{\log n} \leqq \frac{1-\Lambda}{\Lambda} \leqq \limsup _{n \rightarrow \infty} \frac{\log \rho_{n}}{\log n} .
$$

THEOREM C [92]. If $\left\{\alpha_{n}\right\}_{n=0}^{\infty}$ is a sequence of complex numbers of modulus not exceeding one and if $f(z)$ is an entire function of exponential type less than $\log 2$, then $f(z)$ vanishes identically if $f^{(n)}\left(\alpha_{n}\right)=0, n=0,1,2, \cdots$.

We give improved versions of these theorems. Let us denote by $v(r)$ $(0<r<+\infty)$ the central index of the series $f(z)=\sum_{0}^{\infty} a_{n} z^{n}$ for $|z|=r$. Then

$$
\left|a_{n}\right| r^{n} \leqq\left|a_{v(r)}\right| r^{\nu(r)}, \quad n=0,1,2, \cdots
$$

Let

$$
\begin{gathered}
\limsup _{r \rightarrow \infty} \frac{v(r)}{r}=\gamma, \\
\liminf _{r \rightarrow \infty} \frac{v(r)}{r}=\delta .
\end{gathered}
$$

Then we have [85]

$$
\begin{gathered}
\liminf _{n \rightarrow \infty} \frac{\log \max \left(1, n \rho_{n}\right)}{\log n} \leqq \frac{1}{\Lambda}, \\
\frac{1-\lambda}{\lambda} \leqq \limsup _{n \rightarrow \infty} \frac{\log \rho_{n}}{\log n},
\end{gathered}
$$

$$
\frac{\log 2}{\delta} \leqq \limsup _{n \rightarrow \infty} \rho_{n}
$$


and

$$
\liminf _{n \rightarrow \infty} n^{\Lambda-1} \rho_{n}^{\Lambda} \leqq \frac{4^{\Lambda}}{\Lambda T}
$$

Hence if $\Lambda>1, \liminf _{n \rightarrow \infty} \rho_{n}=0$ and if $\Lambda=1$, then since $\delta \leqq t^{*}$ $\left(=\liminf _{r \rightarrow \infty} \log M(r) / r\right) \leqq T^{*}$,

$$
\frac{\log 2}{t^{*}} \leqq \limsup _{n \rightarrow \infty} \rho_{n} ; \quad \liminf _{n \rightarrow \infty} \rho_{n} \leqq \frac{4}{T^{*}} .
$$

The inequalities (8.5)-(8.6) imply Theorem B and (8.7) implies Theorem A. Theorem C follows immediately from (8.7) since $\rho\left(f^{(n)}\right) \leqq r_{n+1}^{*}$ where $r_{k}^{*}$ denotes the absolute value of the zero $z_{k}^{*}$ of $f^{(k)}$ which is nearest to the origin. (If $f^{(k)}$ has no zero then $r_{k}^{*}=\infty$.)

For entire functions defined by gap power series, (8.6) and (8.7) give, in general, better results than Theorems A-C. Let

$$
f(z)=\sum a_{n_{k}} z^{n_{k}} \quad\left(a_{n_{k}} \neq 0, k=1,2, \ldots\right),
$$

be a transcendental entire function and suppose that

$$
\liminf _{k \rightarrow \infty} \log n_{k} / \log n_{k+1}=\chi<1 .
$$

Then $\lambda \leqq \Lambda \chi$ [93] and (8.5) and (8.6) give more information than Theorem B. If we suppose now that $\Lambda \geqq 1$ but $\Lambda \chi<1$ then $\lambda<1, \delta=0$ and (8.7) implies that $\lim \sup _{n \rightarrow \infty} \rho_{n}=\infty$. Thus Theorems $\mathrm{A}$ and $\mathrm{C}$ hold for every function $f$, of any finite order $\Lambda$ and of the form (8.10) with gaps satisfying the condition (8.11) and $\Lambda \chi<1$.

If $f(z)=\sum_{0}^{\infty} a_{n} z^{n}$ and $\left|a_{n} / a_{n+1}\right|$ is ultimately a nondecreasing function of $n$, tending to $\infty$, then $f$ is entire and [85]

$$
\begin{aligned}
& \frac{\log 2}{\gamma} \leqq \liminf _{n \rightarrow \infty} \rho_{n} \leqq \frac{4}{\gamma} \\
& \frac{\log 2}{\delta} \leqq \limsup _{n \rightarrow \infty} \rho_{n} \leqq \frac{4}{\delta}
\end{aligned}
$$

9. Whittaker constant. Consider again Theorem A and let $\alpha$ be the least upper bound of all numbers which can replace $\log 2$ in Theorem A. Read [71] has shown that $\alpha \geqq 0.7259$. Let $W$ be the least upper bound of numbers which can replace $\log 2$ in Theorem $C$. This number is called the Whittaker constant. It is known that (see [71], [11] and the references given there) $0.7259 \leqq W<0.7378$ but the exact value is unknown. Recently Buckholtz [11] has shown that $\alpha=W$.

A simple example of a function $f$ of order one such that each of $f, f^{\prime}$, $f^{\prime \prime}, \ldots$ has a zero in the closed $\operatorname{disc}|z| \leqq 1$ is $f(z)=\sin (\pi z / 4)-\cos (\pi z / 4)$. 
There exist extremal functions for this problem. In fact Evgrafov (see [11]) has shown that there is an entire function $f$ of exponential type $W$ such that each of $f, f^{\prime}, f^{\prime \prime}, \ldots$ has a zero in the $\operatorname{disc}|z| \leqq 1$.

Mention must be made here of a related result due to Erdös and Renyi [26]. Let $f$ be entire and denote by $x=H(y)$ the inverse function of $y=\log M(x)$. Then

$$
\liminf _{k \rightarrow \infty} \frac{H(k)}{k r_{k}^{*}} \leqq \frac{e}{\log 2} .
$$

10. Functions in $E$. (i) Consider first a function $f$ defined by the power series (2.1) and suppose that $a_{n} \neq 0, n\left|\left(a_{n} / a_{n-1}\right)\right| \leqq \log 2$ for $n=2,3, \cdots$. Then $f$ is entire and it can be shown that $\left(f(z)-a_{0}\right) / a_{1} \in E$.

(ii) We now consider functions with all zeros on a ray. Let $\Omega$ denote the family of transcendental entire functions $f$ of the form

$$
f(z)=z e^{\beta z} \prod_{1}^{N}\left(1-z / z_{k}\right)
$$

where $0 \leqq N \leqq \infty$ (if $N=0$, the product disappears) and (a) all $z_{k}$ have the same argument, (b) $\beta z_{1} \leqq 0$ and (c) $1<\left|z_{1}\right| \leqq\left|z_{2}\right| \leqq \cdots$. If $f \in \Omega$ and is univalent in $D$ then [87]

$$
|\beta|+\sum_{k=1}^{N} \frac{1}{\left|z_{k}\right|-1} \leqq 1 .
$$

In fact (10.2) holds if and only if $f$ is starlike in $D$ and all its derivatives are close-to-convex there. Further, if $\left\{z_{k}^{(1)}\right\}_{k=0}^{N}$ are the zeros of $f^{\prime}$, then $f$ and all its derivatives are univalent in $D$ and map $D$ onto convex domains if and only if [87]

$$
|\beta|+\sum_{k=0}^{N} \frac{1}{\left|z_{k}^{(1)}\right|-1} \leqq 1 .
$$

This result implies that $E \cap \Omega=S \cap \Omega$ and that $f \in E \cap \Omega$ if and only if (10.2) holds.

(For the univalence of an entire function of any order see [61].)

(iii) If all zeros of $f$ do not lie on a ray then some derivative $f^{\prime}, f^{\prime \prime}, \ldots$ may have zeros in the unit disc (e.g., $f(z)=\sin (\pi z / 2) /(\pi / 2))$ and then $f$ will not belong to $E$. If however $f$ is of genus zero, and $f(0)=0, f^{\prime}(0)=1$, and the zeros are widely spaced, then $f \in E$. We shall say that a function $f$ has "fourly-spaced" zeros if

$$
\left|z_{1}\right| \geqq 4, \quad\left|z_{k+1}\right| \geqq 4^{k}\left|z_{k}\right|, \quad k \geqq 1 .
$$


Let

$$
P(z)=\prod_{1}^{\infty}\left(1-z / z_{k}\right), \quad f(z)=z P(z) .
$$

Then [78], $f \in E$. It is possible to improve the constant 4 .

11. Multivalent functions. A function $f$ is said to be $p$-valent in $D$ if it is analytic in $D$, if the equation

$$
f(z)=w
$$

has $p$ distinct roots in $D$ for some particular $w$, and if for each complex $w$, equation (11.1) does not have more than $p$ roots in $D$. The function $f$ is also said to have valence $p$ in $D$. When $p=1, f$ is univalent in $D$.

Goodman [34] considered the sum $(f+g) / 2$ and the product $(f g)^{1 / 2}$ when $f$ and $g$ both belong to $S$ and showed that there exist two pairs of functions $f_{1}, g_{1}$ and $f_{2}, g_{2}$ each function belonging to $S$ such that the sum $\left(f_{1}+g_{1}\right) / 2$ and, the product $\left(f_{2}(z) g_{2}(z)\right)^{1 / 2}=z+\cdots$, both have valence $\infty$ in $D$.

We now define areally mean $p$-valent (a.m.p.v.) functions. Let $p$ be a positive number and denote by $n(w)$ the number of roots of the equation (11.1) in $D$. If $f$ is analytic in $D$ and, for every positive $R$,

$$
\frac{1}{\pi R^{2}} \int_{0}^{2 \pi} \int_{0}^{R} n\left(\rho e^{i \phi}\right) \rho d \rho d \phi \leqq p,
$$

then $f$ is said to be a.m.p.v. in $D$. A condition for $f$ to be a.m.p.v. is as follows. Let

$$
\sum_{1}^{\infty}\left|a_{n}\right|=\bar{S}<\left|a_{0}\right|, \quad \sum_{1}^{\infty} n\left|a_{n}\right|^{2}=A<\infty .
$$

Then $f(z)=\sum_{0}^{\infty} a_{n} z^{n}$ is a.m.p.v. in $D$ for all large $p$ such that ([39], [68])

$$
\left|a_{0}\right|>(A / p)^{1 / 2}+\bar{S} \text {. }
$$

If $f$ is a.m.p.v. in $D$ and is normalized and $p=1$, then $\left|a_{2}\right| \leqq 2$ [89]. A bound on $|f|$ is given by the following theorem due to Cartwright, Spencer and Hayman.

THEOREM [38, p. 31]. Suppose that $f(z)=\sum_{0}^{\infty} a_{n} z^{n}$ is a.m.p.v. in D. Then

$$
M(r, f)<A(p) \mu_{p}(1-r)^{-2 p} \quad(0<r<1),
$$

where $\mu_{p}=\max _{0 \leqq v \leqq p}\left|a_{v}\right|$ and $A(p) \leqq(p+2) 2^{3 p-1} \exp \left(p \pi^{2}+\frac{1}{2}\right)$.

This upper bound on the constant $A(p)$ is due to Jenkins and Oikawa 
[45]. In $\S 5(\mathrm{i})$ we have seen that if $f \in S$ and each $f^{(k)}(k=1,2, \ldots)$ is univalent in $D$ then $f$ is a transcendental entire function of exponential type. This result holds under a less restrictive hypothesis. Suppose $f$ is not a polynomial and each $f^{(k)}(k=0,1, \ldots)$ is a.m.p.v. in $D$. Then [81], $f$ is an entire function of exponential type not exceeding $A(p) e(P+2)^{2 p}(P+1)$ where $P=[p]$ is integer part of $p$. If each $f^{\left(n_{j}\right)}, j=1,2, \ldots$, is a.m.p.v. in $D$ and

$$
\lim _{j \rightarrow \infty}\left(n_{j+1}-n_{j}\right)=\infty, \quad n_{j}=O\left(\sum_{k=1}^{j} \log n_{k}\right),
$$

then also $f$ must be entire.

12. Entire functions of bounded index. Let $f(z)=\sum_{n=0}^{\infty} A_{n}(z-a)^{n}$ be an entire function. Since the coefficients tend to zero, there exists a smallest integer $N_{a} \geqq 0$ such that $\left|A_{N_{a}}\right| \geqq\left|A_{n}\right|$ for all $n$. If the integers $N_{a}$ are all bounded above then $f$ is said to be of bounded index and the smallest integer $N$, such that for all numbers $a, N_{a} \leqq N$, is called the index of $f$ (cf., [55], [36]). This is equivalent to the definition given in $§ 1$. As we pointed out a function of bounded index $N$ is of exponential type not exceeding $N+1$. This result is sharp [76]. Denote the class of all functions of bounded index by $B$. The functions $e^{z}, \sin z, \cos z$ are all in $B$.

The Bessel function $J_{k}(z)$ of integer order $k$ is of index $N$ such that $k \leqq N \leqq 2 k-1$ ([52]; see also [60]). Any entire function $f$ satisfying a linear differential equation [77]

$$
P_{0}(z) \frac{d^{n} f}{d z^{n}}+P_{1}(z) \frac{d^{n-1} f}{d z^{n-1}}+\cdots+P_{n}(z) f=Q(z),
$$

where $P_{j}(j=0,1, \ldots, n)$ and $Q$ are polynomials and $\operatorname{deg} P_{j} \leqq \operatorname{deg} P_{0}$ is in class $B$.

Functions with zeros of arbitrarily large multiplicity are obviously of unbounded index. But there are functions [79] of unbounded index and having simple zeros.

The asymptotic properties of $\log M(r, f)$ do not help to prove the boundedness (or the unboundedness) of the index, except that if $T^{*}=\infty$ then $f \in C B$ (the class of entire functions of unbounded index). In fact if $F$ is any transcendental entire function then there are two entire functions $g \in C B[\mathbf{7 0}]$ and $f \in C E$ (the class of entire functions not belonging to $E$ ) such that

$$
\log M(r, g) \sim \log M(r, F) \sim \log M(r, f) .
$$

For $f$ we simply take $f(z)=F(z)-F^{\prime \prime}(0) z^{2} / 2$ !.

We mentioned in $\S 11$ that there exist functions $f$ and $g$ in $S$ such that $(f+g) / 2$ is not in $S$. Pugh [69] showed that the sum of two functions each in $B$, need not be in $B$. 
The class $B$ is not closed under differentiation. There exists [80] an entire function $F$ in $B$ such that the derivative $F^{\prime}$ is in $C B$. If the derivative $f^{\prime}$ is of bounded index $N_{f^{\prime}}, f$ is also of bounded index $N_{f}$ and $N_{f} \leqq N_{f^{\prime}}+1$ [80].

The functions $P$ and $f$ defined by (10.4) and (10.5) are both in $B$. (Cf. [70]. The constant 5 in [70] has been improved to 4 by Mrs. Amy King in her Ph.D dissertation.) In fact, we have, for all $z$,

$$
\max \left\{|P(z)|,\left|P^{\prime}(z)\right|\right\} \geqq\left|P^{(n)}(z)\right|, \quad n=2,3, \cdots .
$$

Furthermore each $P^{(k)}, k=0,1,2, \ldots$, is of index 1 .

Consider now functions with real zeros $a_{n}$. Suppose $a_{1}>0, a_{n+1}-a_{n}$ $\geqq b_{n}(n \geqq 1)$ where the sequence $\left\{b_{n}\right\}_{1}^{\infty}$ is positive and nondecreasing and $\sum_{1}^{\infty} 1 / n b_{n}<\infty$. Then [82],

$$
f(z)=e^{\alpha z+\beta} \prod_{1}^{\infty}\left(1-z / a_{n}\right)
$$

where $\alpha$ and $\beta$ are any complex numbers, is in $B$. If in (12.2) we assume that $a_{1}>0, a_{n+1} / a_{n} \geqq \gamma>1$, then each $f^{(k)}, k=0,1, \ldots$, is in $B$ [54].

We can consider entire functions $f$ satisfying conditions similar to (1.1) and obtain the conclusion that $f$ must be of exponential type [37], [83].

(a) Let $p \geqq 1$ and

$$
I(l, r)=\left\{\int_{0}^{2 \pi}\left|f^{(l)}\left(r e^{i \theta}\right)\right|^{p} d \theta\right\}^{1 / p} .
$$

Let $c$ be a positive constant. Suppose that there exists a positive integer $N$ (independent of $z$ ) such that for $k=0,1,2, \ldots, N$, the following inequality

$$
\sum_{j=0}^{N} \frac{I(k+j, r)}{j !} \geqq c \sum_{j=N+1}^{\infty} \frac{I(k+j, r)}{j !}
$$

holds for all $z$ with $|z|=r$ sufficiently large. Then $f$ is of exponential type and

$$
T^{*} \leqq 1+2 \log (1+1 / c)+\log (2 N) !
$$

(b) Let $c$ be a positive constant. Suppose that there exist two nonnegative integers $k$ and $N$ (independent of $z$ ) such that $f$ satisfies one of the following, for all $z$ with $|z|$ sufficiently large:

$$
\begin{gathered}
\sum_{j=0}^{N} \frac{\left|f^{(k+j)}(z)\right|}{j !} \geqq c \sum_{j=N+1}^{\infty} \frac{\left|f^{(k+j)}(z)\right|}{j !}, \\
\sum_{j=0}^{N} \frac{M\left(r, f^{(k+j)}\right)}{j !} \geqq c \sum_{j=N+1}^{\infty} \frac{M\left(r, f^{(k+j)}\right)}{j !},
\end{gathered}
$$


then $f$ is of exponential type and

$$
T^{*} \leqq \max \left\{N, \min _{1 \leqq j \leqq N}\left(\frac{(N+j) !(N+1)}{(N !) c}\right)^{1 / j},\left(\frac{(2 N+1) !}{(N !) c}\right)^{1 /(N+1)}\right\} .
$$

13. The space of entire functions. Following Iyer [43] we define a metric on the space of all entire functions $\Gamma$. (This space includes all polynomials and constant zero.) Let $f(z)=\sum_{0}^{\infty} a_{n} z^{n}$ and $g(z)=\sum_{0}^{\infty} b_{n} z^{n} \in \Gamma$ and define

$$
d(f, g)=\sup \left\{\left|a_{0}-b_{0}\right|,\left|a_{n}-b_{n}\right|^{1 / n}: n=1,2, \ldots\right\} .
$$

Then $d$ is a metric and $(\Gamma, d)$ is a complete metric space [43]. Let

$$
B_{n}=\{f \in(\Gamma, d) \mid f \text { is of index not exceeding } n\} .
$$

We consider $B=\bigcup_{n=0}^{\infty} B_{n}$ as a subspace of $(\Gamma, d)$. It can be shown that [25] $B_{n}$ is nowhere dense in $B$ and thus $B$ is of the first category.

14. Some applications to summability methods. Let $f$ be entire and $\left\{z_{i}\right\}_{i=0}^{\infty}$ a sequence of complex numbers. We define the matrix transformation $A\left(f, z_{i}\right)=\left(a_{n, k}\right)$ by

$$
f(z)=\sum_{k=0}^{\infty} a_{n, k}\left(z-z_{n}\right)^{k} \text { for } n=0,1, \cdots
$$

We now state some recent results of Fricke and Powell.

I [28]. If $f \in B$ then $A\left(f, z_{i}\right)=\left(a_{n, k}\right)$ is not regular for any sequence $\left\{z_{i}\right\}_{i=0}^{\infty}$. (A transformation $A=\left(a_{n, k}\right)$ is regular if it transforms every convergent sequence into a sequence converging to the same limit. See [41, p. 43].)

Define a sequence $\left\{a_{n}\right\}_{0}^{\infty}$ to be entire if $f(z)=\sum_{0}^{\infty} a_{n} z^{n}$ is an entire function. An entire sequence $\left\{a_{n}\right\}_{0}^{\infty}$ is said to be a sequence of bounded index if $f(z)=\sum_{0}^{\infty} a_{n} z^{n} \in B$. We denote by $\varepsilon$ the set of all entire sequences and by $\mathscr{B}$ the set of all entire sequences of bounded index. An infinite matrix $A=\left(a_{n, k}\right)$ of complex numbers which transforms $\varepsilon$ into $\varepsilon$ is said to be an $\varepsilon-\varepsilon$ method (entire method).

II [27]. A matrix $A=\left(a_{n, k}\right)$ is an $\varepsilon-\varepsilon$ method if and only if for each integer $q>0$, there exists an integer $p>0$ and a constant $M>0$ such that

$$
\left|a_{n, k}\right| q^{n} \leqq M p^{k} \quad \text { for all } n, k=0,1, \cdots .
$$

Let $A^{\prime}\left(f, z_{i}\right)=\left(b_{n, k}\right)$ denote the transpose of $A\left(f, z_{i}\right)=\left(a_{n, k}\right)$, that is, $b_{n, k}=a_{k, n}$.

III [28]. If $f \in B$ then for any sequence $\left\{z_{i}\right\}_{i=0}^{\infty}, A^{\prime}\left(f, z_{i}\right)=\left(b_{n, k}\right)$ is an $\varepsilon-\varepsilon$ method if and only if for each integer $n>0$ there exist an integer $p>0$ and a constant $M>0$ such that

$$
\left|f^{(n)}\left(z_{k}\right)\right| \leqq p^{k} M \quad \text { for } k=0,1, \cdots .
$$


The condition that $f \in B$ is essential in III.

We now define the $l-l$ method. Let $s$ be the set of all sequences of complex numbers. Let

$$
l=\left\{x=\left\{x_{n}\right\}_{n=0}^{\infty} \in S\left|\sum_{0}^{\infty}\right| x_{n} \mid<\infty\right\} .
$$

A matrix $A=\left(a_{n, k}\right)$ that maps $l$ into itself is said to be an $l-l$ method. Knopp and Lorentz [49] proved that a matrix $A=\left(a_{n, k}\right)$ is an $l-l$ method if and only if there exists a constant $M>0$ such that

$$
\sum_{n=0}^{\infty}\left|a_{n, k}\right| \leqq M \quad \text { for } k=0,1, \cdots
$$

IV [28]. Let $f \in B$ and $\left\{z_{i}\right\}_{i=0}^{\infty}$ be a sequence of complex numbers. If either $A\left(f, z_{i}\right)=\left(a_{n, k}\right)$ or $A^{\prime}\left(f, z_{i}\right)=\left(b_{n, k}\right)$ is an $l-l$ method then $A^{\prime}\left(f, z_{i}\right)$ is an $\varepsilon-\varepsilon$ method.

Finally we give a matrix which transforms $\mathscr{B}$ into $\mathscr{B}$.

Let the Taylor matrix $T(\xi)=\left(a_{n, k}\right)$ be defined by

$$
\begin{aligned}
a_{n, k} & =\left(\begin{array}{l}
k \\
n
\end{array}\right)(1-\xi)^{n+1} \xi^{k-n}, & & \text { if } k \geqq n, \\
& =0, & & \text { otherwise },
\end{aligned}
$$

where $\xi$ is a complex number.

$\mathrm{V}$ [28]. The Taylor matrix $T(\xi)=\left(a_{n, k}\right)$ transforms $\mathscr{B}$ into $\mathscr{B}$ for any complex number $\xi$.

15. Conjectures and open problems. We now list some problems and conjectures connected with two classes $E$ and $B$.

CONJECTURE 1. If $\phi$ is any transcendental entire function such that

$$
\limsup _{r \rightarrow \infty} \frac{\log M(r, \phi)}{r} \leqq \pi
$$

there exists an entire function $f \in E$ such that $\log M(r, \phi) \sim \log M(r, f)$ $(r \rightarrow \infty)$.

CONJECTURE 2. If $\phi$ is any entire function of exponential type, there exists an entire function $f \in B$ such that $\log M(r, \phi) \sim \log M(r, f)(r \rightarrow \infty)$.

For some theorems of this type, but not connected with $E$ or $B$, see [22], [19], [20].

Conjecture 3. $W=2 / e$.

ConjeCtURE 4. If $\sum_{p=1}^{\infty} 1 / n_{p}=\infty$ and $\rho\left(f^{\left(n_{p}\right)}\right) \geqq 1$ for $p=1,2, \ldots$, then $f$ is entire.

In the following problems $1-4, f \in E$. 
1. What is the smallest zero that $f$ can have? (Exclude $z=0$.)

2. What is the largest circle center origin covered by $f(D)$ ?

3. Find bounds on $\left|a_{2}^{2}-a_{3}\right|$.

4. Find $\alpha=\sup \left\{\left|a_{2}\right| \mid f \in E\right\}$.

5. Find $\alpha_{K}=\sup \left\{\left|a_{2}\right| \mid f \in T(K)\right\}$.

6. Let $f$ be entire and satisfy a differential equation of the form (12.1). Assume $P_{j}(j=0,1, \ldots, n)$ and $Q$ are polynomials and $\operatorname{deg} P_{j} \leqq \operatorname{deg} P_{0}$. Then $f$ is of bounded index. Find an estimate for the index.

\section{BIBLIOGRAPHY}

1. D. Aharonov, Proof of the Bieberbach conjecture for a certain class of univalent functions, Israel J. Math. 8 (1970), 102-104. MR 42 \# 486.

2. J. Becker, Über Subordinationsketten und quasikonform fortsetzbare schlichte Funktionen, Ph.D. Thesis, Technische Universität, Berlin, 1970.

3. S. D. Bernardi, $A$ survey of the development of the theory of schlicht functions, Duke Math. J. 19 (1952), 263-287. MR 14, 35.

4. - A bibliography of schlicht functions, New York University, Courant Institute of Mathematical Sciences, New York, 1966.

5. - The radius of univalence of certain analytic functions, Proc. Amer. Math. Soc. 24 (1970), 312-318.

6. L. Bieberbach, Über die Koeffizienten derjenigen Potenzreihen, welche eine schlichte Abbildung des Einheitkreises vermitteln, S.-B. Preuss. Akad. Wiss. 138 (1916), 940-955.

7. R. P. Boas, Univalent derivatives of entire functions, Duke Math. J. 6 (1940), 719-721. MR 2, 82.

8. Entire functions, Academic Press, New York, 1954. MR 16, 914.

9. E. Bombieri, On the local maximum property of the Koebe function, Invent. Math. 4 (1967), 26-67. MR 36 \#1635.

10. D. A. Brannan, J. Clunie and W. E. Kirwan, Coefficient estimates for a class of star-like functions, Canad. J. Math. 22 (1970), 476-485. MR 41 \# 5614.

11. J. D. Buckholtz, The Whittaker constant and successive derivatives of entire functions, J. Approximation Theory 3 (1970), 194-212. MR 41 \# 5618.

12. - Successive derivatives of analytic functions, Indian J. Math. (to appear).

13. J. D. Buckholtz and J. L. Frank, Whittaker constants, Proc. London Math. Soc. (3) 23 (1971), 348-370.

14. D. M. Campbell, J. A. Cima and J. A. Pfaltzgraff, Linear spaces and linear-invariant families of locally univalent analytic functions, Manuscripta Math. 4 (1971), 1-30.

15. J. A. Cima and J. A. Pfaltzgraff, A Banach space of locally univalent functions, Michigan Math. J. 17 (1970), 321-334.

16. W. M. Causey, The close-to-convexity and univalence of an integral, Math. Z.99 (1967), 207-212. MR 35 \#6807.

17. W. M. Causey and E. P. Merkes, Radii of starlikeness of certain classes of analytic functions, J. Math. Anal. Appl. 31 (1970), 579-586. MR 41 \#8644.

18. Z. Charzyński and M. Schiffer, A new proof of the Bieberbach conjecture for the fourth coefficient, Arch. Rational Mech. Anal. 5 (1960), 187-193. MR 22 \# 5746.

19. J. Clunie, On integral functions having prescribed asymptotic growth, Canad. J. Math. 17 (1965), 396-404. MR 31 \#350.

20. J. Clunie and T. Kövari, On integral functions having prescribed asymptotic growth. II, Canad. J. Math. 20 (1968), 7-20. MR 37 \# 2995.

21. V. F. Cowling and W. C. Royster, Domains of variability for univalent polynomials, Proc. Amer. Math. Soc. 19 (1968), 767-772. MR 37 \# 2976.

22. A. Edrei and W. H. J. Fuchs, Entire and meromorphic functions with asymptotically prescribed characteristic, Canad. J. Math. 17 (1965), 383-395. MR 30 \# 4942.

23. P. Eenigenburg, On the radius of curvature for convex analytic functions, Canad. J. Math. 22 (1970), 486-491. MR 41 \# 5608.

24. P. J. Eenigenburg and F. R. Keogh, The Hardy class of some univalent functions and their derivatives, Michigan Math. J. 17 (1970), 335-346.

25. K. A. Ekblaw, The functions of bounded index as a subspace of a space of entire functions, Pacific J. Math. 37 (1971), 353-355. 
26. P. Erdös and A. Rényi, On the number of zeros of successive derivatives of analytic functions, Acta Math. Acad. Sci. Hungar. 7 (1956), 125-144. MR 18, 201.

27. G. H. Fricke and R. E. Powell, A theorem on entire methods of summation, Compositio Math. 22 (1970), 253-259.

28. - Functions of bounded index and summability methods (to appear).

29. S. Friedland and Z. Nehari, Univalence conditions and Sturm-Liouville eigenvalues, Proc. Amer. Math. Soc. 24 (1970), 595-603. MR 40 \# 4435.

30. P. R. Garabedian, G. G. Ross and M. M. Schiffer, On the Bieberbach conjecture for even $n$, J. Math. Mech. 14 (1965), 975-989. MR 32 \#207.

31. P. R. Garabedian and M. M. Schiffer, The local maximum theorem for the coefficients of univalent functions, Arch. Rational Mech. Anal. 26 (1967), 1-32. MR 37 \# 1584.

32. G. M. Goluzin, Geometric theory of functions of a complex variable, GITTL, Moscow, 1952; English transl., Transl. Math. Monographs, vol. 26, Amer. Math. Soc., Providence, R.I., 1969. MR 15, 112; MR 40 \# 308.

33. A. W. Goodman, Univalent functions and nonanalytic curves, Proc. Amer. Math. Soc. 8 (1957), 598-601. MR 19, 260.

34. $\ldots$, The valence of sums and products, Canad. J. Math. 20 (1968), 1173-1177.

MR 38 \# 314

35. , Open problems on univalent and multivalent functions, Bull. Amer. Math. Soc. 74 (1968), 1035-1050. MR 38 \#315.

36. Fred Gross, Entire functions of bounded index, Proc. Amer. Math. Soc. 18 (1967), 974-980. MR 36 \# 1649.

37. ㄴ. Entire functions of exponential type, J. Res. Nat. Bur. Standards Sect. B 74B (1970), 55-59. MR 42 \# 501.

38. W. K. Hayman, Multivalent functions, Cambridge Tracts in Math. and Math. Phys., no. 48, Cambridge Univ. Press, Cambridge, 1958. MR 21 \# 7302.

39. - Coefficient problems for univalent functions and related function classes, J. London Math. Soc. 40 (1965), 385-406; Corrigendum, ibid. 41 (1966), 550. MR 31 \# 3592; MR 33 \# 7519.

40. — Research problems in function theory, Athlone Press, London, 1967. MR 36 \# 359 .

41. G. H. Hardy, Divergent series, Clarendon Press, Oxford, 1949. MR 11, 25.

42. J. A. Hummel, The coefficients of starlike functions, Proc. Amer. Math. Soc. 22 (1969), 311-315. MR 40 \# 4440.

43. V. G. Iyer, On the space of integral functions. I, J. Indian Math. Soc. 12 (1948), 13-30. MR 10, 380.

44. Z. J. Jakubowski, Sur les coefficients des fonctions univalentes dans la cercle unité, Ann. Polon. Math. 19 (1967), 207-233. MR 35 \#6814.

45. J. A. Jenkins and K. Oikawa, On results of Ahlfors and Hayman, Illinois J. of Math. (to appear).

46. W. Kaplan, Close-to-convex schlicht functions, Michigan Math. J. 1 (1952), 169-185. MR 14, 966.

47. F. R. Keogh, A strengthened form of the 1/4 theorem for starlike univalent functions,

Macintyre Memorial Volume, Ohio Univ. Press, Athens, Ohio, 1970, pp. 201-211.

48. A. C. King and S. M. Shah, Indices of Lindelöf functions and their derivatives, Rocky

Mt. J. Math. (to appear).

49. K. Knopp and G. G. Lorentz, Beiträge zur absoluten Limitierung, Arch. Math. 2 (1949), 10-16. MR 11, 346.

50. J. G. Krzyż, On close-to-convex functions, Symposia on Theoretical Physics and Math., vol. 10 (Inst. Math. Sci., Madras, 1969), Plenum, New York, 1970, pp. 23-27. MR 41 \# 5610

51. Boo-sang Lee and S. M. Shah, The type of an entire function satisfying a linear differential equation, Arch. Math. (Basel) 20 (1969), 616-622. MR 41 \# 544.

52., An inequality involving the Bessel function and its derivatives, J. Math. Anal. Appl. 30 (1970), 144-156. MR 41 \#527.

53. - Inequalities satisfied by entire functions and their derivatives, Trans. Amer. Math. Soc. 149 (1970), 109-117. MR 41 \# 2006.

54. - On the growth of entire functions of bounded index, Indiana Univ. Math. J. 20 (1970/71), 81-87. MR 41 \# 3766.

55. B. Lepson, Differential equations of infinite order, hyperdirichlet series and entire functions of bounded index, Proc. Sympos. Pure Math., vol. XI, Amer. Math. Soc., Providence, R.I., 1968, pp. 298-307. MR 38 \#6069.

56. N. Levinson, $A$ theorem of Boas, Duke Math. J. 8 (1941), 181-182. MR 2, 274. 
57. R. J. Libera, Some classes of regular univalent functions, Proc. Amer. Math. Soc. 16 (1965), 755-758. MR 31 \#2389.

58. K. Löwner, Untersuchungen über schlichte konforme Abbildungen des Einheitkreises. I, Math. Ann. 89 (1923), 103-121.

59. T. H. MacGregor, Univalent power series whose coefficients have monotonic properties, Math. Z. 112 (1969), 222-228. MR 40 \# 2839.

60. V. Marić and S. M. Shah, Entire functions defined by gap power series and satisfying a differential equation, Tôhoku Math. J. (2) 21 (1969), 621-631. MR 41 \# 3763.

61. E. P. Merkes, M. S. Robertson and W. T. Scott, On products of starlike functions, Proc. Amer. Math. Soc. 13 (1962), 960-964. MR 26 \# 316.

62. Z. Nehari, The Schwarzian derivative and schlicht functions, Bull. Amer. Math. Soc. 55 (1949), 545-551. MR 10, 696.

63. - Conformal mapping, McGraw-Hill, New York, 1952. MR 13, 640.

64. M. Ozawa, On the Bieberbach conjecture for the sixth coefficient, Ködai Math. Sem. Rep. 21 (1969), 97-128. MR 39 \#432. An elementary proof of the Bieberbach conjecture for the sixth coefficient, Kōdai Math. Sem. Rep. 21 (1969), 129-132. MR 40 \# 333.

65. K. S. Padmanabhan, On the radius of univalence of certain classes of analytic functions,

J. London Math. Soc. (2) 1 (1969), 225-231. MR 40 \# 331.

66. R. N. Pederson, A proof of the Bieberbach conjecture for the sixth coefficient, Arch.

Rational Mech. Anal. 31 (1968/69), 331-351. MR 39 \#431.

67. G. Pólya, On the zeros of the derivatives of a function and its analytic character, Bull. Amer. Math. Soc. 49 (1943), 178-191. MR 4, 192.

68. C. Pommerenke, Über die Mittlewerte und Koeffizienten multivalenter Funktionen, Math. Ann. 145 (1961/62), 285-296. MR 24 \# A3282.

69. W. Pugh, Sums of functions of bounded index, Proc. Amer. Math. Soc. 22 (1969), 319-323. MR 39 \# 4391.

70. W. J. Pugh and S. M. Shah, On the growth of entire functions of bounded index, Pacific J. Math. 33 (1970), 191-201. MR 41 \# 3767.

71. G. A. Read, Univalent derivatives of entire functions, J. London Math. Soc. (2) 1 (1969), 189-192. MR 40 \#2860.

72. , Related interpolation problems, J. London Math. Soc. (2) 2 (1970), 353-357. MR 41 \# 8882.

73. M. S. Robertson, Quasi-subordination and coefficient conjectures, Bull. Amer. Math. Soc. 76 (1970), 1-9. MR 40 \# 4441.

74. ㄴ. The sum of univalent functions, Duke Math. J. 37 (1970), 411-419. MR 41 \#8649

75. W. C. Royster, On the univalence of a certain integral, Michigan Math. J. 12 (1965), 385-387. MR 32 \# 1342.

76. S. M. Shah, Entire functions of bounded index, Proc. Amer. Math. Soc. 19 (1968), 1017-1022. MR 38 \#6070.

77. - Entire functions satisfying a linear differential equation, J. Math. Mech. 18 (1968/69), 131-136. MR 37 \#2994.

78. Univalent derivatives of entire functions of slow growth, Arch. Rational Mech. Anal. 35 (1969), 259-266. MR 40 \#352.

79. ㄴ. Entire functions of unbounded index and having simple zeros, Math. Z. 118 (1970), 193-196.

80. - On entire functions of unbounded index whose derivatives are of unbounded index, J. London Math. Soc. (2) 4 (1971), 127-139.

81. Holomorphic functions with mean p-valent derivatives, Math. Ann. 193 (1971), $176-182$.

82. S. M. Shah and S. N. Shah, A new class of functions of bounded index (to appear).

83. S. M. Shah and W. C. Sisarcick, On entire functions of exponential type, J. Res. Nat. Bur. Standards Sect. B (to appear).

84. S. M. Shah and S. Y. Trimble, Univalent functions with univalent derivatives, Bull. Amer. Math. Soc. 75 (1969), 153-157. MR 39 \#433; Erratum, ibid, 75 (1969), 888; MR 39 \#4373.

85. Univalent functions with univalent derivatives. II, Trans. Amer. Math. Soc. 144 (1969), 313-320. MR 40 \# 2841.

86. - Univalent functions with univalent derivatives. III, J. Math. Mech. 19(1969/70), 451-460. MR 40 \# 4438.

87. - Entire functions with univalent derivatives, J. Math. Anal. Appl. 33 (1971), 220-229; Errata, ibid, 35 (1971), 692. 
88. V. Singh, Grunsky inequalities and coefficients of bounded schlicht functions, Ann. Acad. Sci. Fenn. Ser. A I No. 310 (1962), 22 pp. MR 26 \# 1444.

89. D. C. Spencer, On mean one-valent functions, Ann. of Math. (2) 42 (1941), 614-633. MR 3, 78.

90. T. J. Suffridge, A coefficient problem for a class of univalent functions, Michigan Math.

J. 16 (1969), 33-42. MR 39 \# 1646.

91. - Some remarks on convex maps of the unit disk, Duke Math. J. 37 (1970), $775-777$.

92. S. Takenaka, On the expansion of integral transcendental functions in generalized Taylor series, Proc. Physics Math. Soc. Japan 14 (1932), 529-542.

93. J. M. Whittaker, The lower order of integral functions, J. London Math. Soc. 8 (1933), 20-27.

94. D. J. Wright, The radius of p-valent starlikeness for certain classes of analytic functions, Ann. Polon. Math. 23 (1970/71), 49-55.

95. M. R. Ziegler, Some integrals of univalent functions, Indian J. Math. 11 (1969), 145-151. MR 41 \#3743.

Department of Mathematics, University of Kentucky, LeXington, Kentucky 40506 